\title{
DISCURSOS EN CONTIENDA: TAMBIÉN LA LLUVIA DE ICÍAR BOLLAÍN
}

\author{
POR \\ Myriam OsORIO \\ Memorial University of Newfoundland
}

También la lluvia, el quinto largometraje de la directora española Icíar Bollaín, ofrece una singular mirada al presente y al pasado de América. ${ }^{1}$ Estrenada en el año 2010 y filmada en Bolivia, la cinta se compone de dos relatos intercalados: el rodaje de una película en Cochabamba sobre la llegada de Cristobal Colón al nuevo mundo en 1492, cuyo resultado fue el sometimiento de la población indígena, y las guerras del agua que ocurren en el país andino en abril del año 2000. ${ }^{2}$ Bollaín incorpora la tensa situación que se vive en Bolivia a manera de película dentro de la película para subrayar la persistencia del legado colonial en sus re-encarnaciones a comienzos del siglo XXI y la resistencia popular a las nuevas presiones. Los personajes del primer relato incluyen figuras como Cristobal Colón (Karra Elejalde), Bartolomé de las Casas (Carlos Santos), Antonio de Montesinos (Raúl Arévalo), el líder indígena Hatuey (Juan Carlos Aduviri) y un gran número de extras. En el relato paralelo participan los actores que representan a los anteriores personajes, además del productor Costa (Luis Tosar), el director Sebastián (Gael García Bernal) y el activista Daniel (Aduviri). En su reseña sobre la película Jorge Marí señala que en ella "[...] se entremezclan dos niveles -el de la representación histórica [...] y el de la realidad boliviana contemporánea-, de manera que cada una de las narraciones funciona como un espejo de la otra, y varios

1 Entre los anteriores cabe mencionar, Flores de otro mundo (1999), Te doy mis ojos (2003) y Mataharis (2007). La directora se desempeñó como actriz en Land and Freedom (1995), cinta sobre la Guerra Civil española, del director británico Ken Loach. También la lluvia es una coproducción entre España, Francia y México y ha recibido varios premios: el Ariel en México a la mejor película Iberoamericana y tres Goya: mejor música original de Alberto Iglesias, mejor dirección de producción para Cristina Zumárraga y mejor actor de reparto para Karra Elejalde en el año 2011. Ese mismo año recibió la nominación española para los premios Óscar en la categoría de mejor película extranjera.

2 El nombre Cochabamba proviene de las palabras del quechua khucha, pantanoso, y pampa llanura, que significa llanura pantanosa. La ciudad está ubicada en el centro de Bolivia, es la capital del Departamento de Cochabamba y un importante centro agrícola. En los años 70 hubo corrientes de relocalización de los sectores mineros, que se ubicaron en las zonas periféricas de la ciudad. 
de los personajes (muy especialmente Daniel/Hatuey [...]) se mueven simultáneamente en dos esferas espacio-temporales" (369).

La correlación entre los dos relatos que corren paralelos, unida a la dedicatoria de la película a la memoria de Howard Zinn, ${ }^{3}$ reconocido historiador y activista comprometido en movimientos de justicia social y en presentar la historia y la voz de "la gente", permiten enfocar el análisis de la película en torno a los siguientes interrogantes: ¿Quién posee el derecho a hablar? ¿Qué dice? ¿Cómo se cuenta la historia de la gente? ¿Qué perspectiva se valora? ¿Qué efecto (ideológico) tiene dicha valoración en el intento de re-escribir la Historia? ¿Cómo contribuye la técnica cinematográfica a explorar estos interrogantes? Estas preguntas son relevantes en cuanto También la lluvia proyecta una multiplicidad de personajes cuyos discursos están en tensión: el del productor, el del director, el discurso histórico de la colonización, el de Hatuey/Daniel y el de las compañías multinacionales. Estas perspectivas dispares convergen en Hatuey/Daniel, personaje quien a través de su voz contestataria obtiene amplio espacio representativo y discursivo en la cinta. En También la lluvia la voz indígena suprimida durante la colonización resurge con fuerza en el siglo XXI para cuestionar el legado histórico de la misma y rechazar el discurso hegemónico de las corporaciones multinacionales que pretenden privatizar el agua.

La expresión “discurso" se emplea aquí en el sentido que le adscribe Michel Foucault: se trata de un sistema de representación que genera significados en un contexto histórico. ${ }^{4}$ El discurso puede ser escrito u oral y a través de él se realizan numerosas operaciones: definir y ubicar a los personajes en una escala o posición social; producirlos y permitir que se produzcan a sí mismos a través del lenguaje, entre otros aspectos. El discurso tiene ingerencia tanto en las relaciones de poder (entre individuos y entre los individuos y el Estado), como en las posibilidades de agencia. Según Stuart Hall, para Foucault "Discourse [...] never consists of one statement, one text, one action or one source. The same discourse, characteristic of the way of thinking or the state of knowledge at any one time [...], will appear across a range of texts, and as forms of conduct, at a number of different institutional sites within society" (72-73). Además de lo anterior, Foucault también reconoce que "[...] that a different discourse [...] will arise at a later historical moment, supplanting the existing one, opening up a new discursive formation, and producing $[\ldots]$ new conceptions $[\ldots]$, new discourses with the power and authority $[\ldots]$, to regulate social practices in new ways" (74). En otras palabras, el discurso de la historia es el discurso del poder, que busca controlar el conocimiento, las instituciones y

3 Howard Zinn es autor de más de 20 libros que versan sobre múltiples temas, entre ellos, la lucha por los derechos civiles y la oposición a las guerras (Vietnam, Irak). Su volumen más conocido es A People's History of the United States (1980).

4 Cabe mencionar que dichos discursos son el resultado de un libreto, compuesto (y negociado con la directora) por Paul Laverty.

\footnotetext{
Revista Iberoamericana, Vol. LXXXI, Núm. 251, Abril-Junio 2015, $523-537$ 
a los individuos en una sociedad. No obstante, Foucault propone la necesidad de buscar las rupturas del discurso para identificar los fragmentos de la historia excluidos por los poderes políticos y sociales. La estructura pendular de También la lluvia (TLL) registra ciertas maneras de pensar y su aparición a través del tiempo y el espacio. Empero, también busca las rupturas, las voces olvidadas para cuestionar el discurso autorizado y transformarlo, re-escribirlo.

Como sistema que se compone de múltiples discursos y de complejas capas de representación a nivel de la narrativa y de la técnica, el cine es un medio excepcional para crear y cuestionar significados. Estos dos propósitos se manifiestan, entre otros, en el manejo del sonido diegético: ${ }^{5}$ el lenguaje (discurso) que utilizan los personajes para comunicar sus luchas cotidianas, sus ideas y para mostrar los fuertes conflictos que incitan inevitablemente a producir discursos de resistencia. En Audio-Vision, un estudio sobre el sonido en la pantalla cinematográfica, Michel Chion sostiene que el cine es un fenómeno "verbocéntrico" (5). Chion se refiere a que los seres humanos en su comportamiento habitual son "voco-and verbocentric"(6); es decir, que como seres sociales producen discurso a través del lenguaje y la pantalla es un medio idóneo para representar dicho comportamiento. Las ideas de Chion, conectadas a las de Foucault, recalcan que lo que se dice puede producir efectos de mínimas o amplias proporciones. En También la lluvia el efecto del discurso es poderoso porque representa derrotas y victorias en una dialéctica constante para contar "lo que pasó".

Para contar lo que pasó, el discurso de TLL se ajusta y se desvía del discurso historiográfico autorizado. Se desvía al situar en Bolivia la llegada de Colón, un hecho crucial que se desarrolló en el Caribe americano. Esta yuxtaposición pone de manifiesto (y reitera) la inevitable imbricación entre la ficción fílmica y el discurso histórico. El productor y el director son concientes de la discrepancia en la que incurren al Sebastián plantear la pregunta: ¿qué hace Colón entrevistándose con indígenas de los Andes? Aunque la pregunta subraya el carácter verbocéntrico (entrevistándose) de la relación entre las dos partes, la respuesta radica en la lógica comercial (que también utilizara Colón) de máximo provecho con un mínimo de inversión económica. Costa quiere filmar su película en exteriores, lo cual le resulta más barato en Bolivia, ${ }^{6}$ país que para él está "lleno de indígenas hambrientos" a quienes va a emplear como extras. El papel de extras implica, entre otras cosas, que los indígenas aparecerán, por lo general en el segundo plano del encuadre por algunos segundos para crear la ilusión de realidad,

5 Jordan y Allison definen el sonido diegético de la siguiente manera: "Diegetic Sound has its source in the 'story', normally in the framed image. But diegetic sound can also be off-screen. Of the three types of sound -speech, music and noise- dialogue is normally prioritized, since it often drives the narrative" (48-50).

6 La elección de filmar en exteriores deriva de aspectos importantes, entre ellos el libreto y la necesidad de filmar en zonas rurales como exigencia del film histórico.

Revista Iberoamericana, Vol. LXXXI, Núm. 251, Abril-Junio 2015, 523-537 ISSN 0034-9631 (Impreso)

ISSN 2154-4794 (Electrónico) 
pero permanecerán en silencio. Serán lo que en la jerga cinematográfica se denomina "paisaje humano". Como extras tampoco recibirán reconocimento en los créditos al final de la película, aunque haya una exigua remuneración por su trabajo: dos dólares al día. ${ }^{7}$ Se trata de personas que reciben poco respeto, y esta verdad no se oculta en la película porque Costa las ubica en una posición social muy inferior a la suya. En este sentido, volviendo a Foucault, el discurso de Costa se ajusta a las ideas en circulación sobre la población indígena; es decir, repite los significados, la manera de pensar y el conocimento que se generaron en el contexto histórico de la colonización y subraya la insidiosa persistencia de esta ideología. Según dicha idelogía, las personas de origen europeo son superiores a la población indígena de las Américas. Costa permite recordar el discurso colombino, en el cual el almirante excluye el discurso indígena y además afirma en la "Relación del primer viaje" que a la gente de la isla les dio "cosas muchas de poco valor con que hobieron mucho placer" (24).

Ahora bien, filmar en Bolivia tiene una significacion más profunda: mostrar que la lógica económica aplicada por Costa, tan similar al modelo colonizador del pasado, busca replicarse o extenderse a todos los sitios donde sea posible. ${ }^{8}$ Así, el resultado devastador de la llegada de Colón a la isla de Guanahani, es fácilmente observable en otras latitudes. La dinámica de adaptación y contravención adoptada por Bollaín expone las contradicciones y busca las posibilidades de transformación. En este sentido, resulta útil mencionar las ideas de Robert A. Rosenstone sobre el cometido de la historia y el cine en la posmodernidad: "[...] they refer to actual events, moments, and movements from the past, and at the same time they partake of the unreal and the fictional, since both are made out of sets of conventions we have developed for talking about where human beings have come from (and also where we are and where we think we are going [...])" (2).

Para reforzar la relación del pasado histórico con el presente, el comienzo de También la lluvia presenta la perspectiva de los extranjeros que llegan a una zona periférica de Cochabamba a buscar no solamente los extras, sino también a alguien que encarne al líder indígena Hatuey. La focalización de la cámara, en toma de primer plano, se centra en Sebastián, el director sorprendido al ver una larga fila de campesinos e indígenas, mujeres, hombres, niñas y niños que acuden a pie al llamado para participar en un "casting abierto". La agenda de Costa es mantener los parámetros del trato puramente comercial y por lo tanto reducir a un mínimo la interacción con las personas que esperan.

7 La lista de créditos al final de También la lluvia no incluye una categoría destinada a los extras. No obstante, contiene agradecimientos al Gobierno de Bolivia, a la Presidencia de la Nación, al Ministerio de Cultura, la población y vecinos de Villatunari, a las seis Federaciones de Campesinos y Cocaleros del Trópico Cochabamba y a los hombres y mujeres del pueblo de Cochabamba.

8 Especialmente después de la caída del muro de Berlín y la consolidación de la democracia liberal y el capitalismo como únicas alternativas para el mundo.

\footnotetext{
Revista Iberoamericana, Vol. LXXXI, Núm. 251, Abril-Junio 2015, $523-537$ 
Por lo tanto le pide a Sebastián que escoja "los que te gusten", y a los demás, insiste, "los echas". Sebastián, sin embargo, no logra cumplir con esa orden. Aunque la multitud que espera parece silenciosa y sumisa, dicha sumisión se transforma rápidamente en protesta colectiva cuando los responsables del "casting" les piden que se vayan porque (les dicen) no pueden "verlos a todos". La voz de protesta es la de Daniel, quien con el apoyo de los demás, los obliga a cumplir con los términos escritos en un volante distribuido en la zona. En este segmento, retomando a Foucault y a Chion, el discurso contrario de Daniel produce agencia individual y colectiva al tiempo que cuestiona las relaciones de poder y control que especialmente Costa (desde su posición de ser la fuente del dinero) desea implantar. Daniel con su lenguaje agresivo, "carajo", "blanquito", "hemos venido caminando desde muy lejos", "de aquí no nos vamos", "nos tienen que ver a todos" realiza una primera ruptura, o una transformación en el proceso de articular la historia: no da espacio para convertirse en otro fragmento excluido y olvidado de la historia. Por otro lado, el discurso de Daniel con su tremenda carga significativa le infunde gran dramatismo a la escena y la dirige hacia un objetivo: la creación de un sentido de inminencia y expectativa (Chion 14). El sentido de inminencia se refuerza al ser Daniel, quien por su carácter aguerrido y marcados rasgos indígenas, consigue el papel del líder indígena Hatuey.

A pesar del estatus, los recursos económicos y la autoridad de los extranjeros, estos se ven forzados a aceptar la voluntad, la voz, de una comunidad que ha visto muchas veces sus derechos ignorados. ${ }^{9}$ Sebastián, concluye entonces declarando: "Tiene razón Daniel. Vamos a ver a todos". Esta secuencia aún con los problemas de intereses ulteriores que plantea, (la realización de película por encima de cualquier otra consideración), y que resultan tal vez imposibles de evitar alinean el proyecto de Bollaín con la visión de Marcia Landy ${ }^{10}$ con respecto a la historiografía tradicional, al señalar que: "[...] too often existing forms of history are disseminated by the victor and therefore deserve to be challenged as well as reexamined so that different conceptions of social and cultural change may be developed" (42). Bollaín quiere mostrar el otro lado, el reto y la posibilidad de un cambio social al desestabilizar la dinámica colonizador/ colonizado y cuestionar el discurso de los vencedores.

Los discursos en contienda de Costa y Daniel construyen de manera implícita al personaje de Cristobal Colón. Ahora bien, dicha construcción se hace también a través de

9 La música original de Alberto Iglesias (sonido extradiegético) que acompaña la escena entona notas andinas tristes, las cuales tienen el efecto de apelar a las emociones de la audiencia para que adopte, en oposición a Costa, el argumento ideológico de la película. Como espectadores, "we should 'feel' for the sufferings of others, put aside selfish feelings or bottom-line business as usual mandates, and help them" (Ryan y Lenos 111).

10 Landy atribuye sus ideas particularmente a pensadores como Walter Benjamin, Michel Foucault, Gilles Deleuze y Felix Guatari.

Revista Iberoamericana, Vol. LXXXI, Núm. 251, Abril-Junio 2015, 523-537 ISSN 0034-9631 (Impreso)

ISSN 2154-4794 (Electrónico) 
otros medios: la espectacular entrada en escena de una enorme cruz oscura, transportada por un helicóptero. ${ }^{11}$ El foco de la cámara, los personajes y los espectadores se dirigen al plano superior para enfocarse tanto en el objeto, como en el sonido ensordecedor que genera su entrada y que apaga el discurso de los que reclaman en el plano inferior. El efecto de hacer enmudecer a la multitud es acorde con la doble misión de cristianizar y colonizar. La poderosa imagen cinematográfica dialoga de manera indirecta con la Carta a Luis de Santángel, en la cual Colón afirma que los indígenas " “...] creían muy firme que yo con estos navíos y gente venía del cielo [...]" (188). Como un objeto valioso traído por los realizadores, que la reciben con gritos de júbilo, la cruz simboliza no solamente sus intereses, sino también los intereses religiosos y políticos de Colón. Como vocero de los Reyes Católicos, Colón deseaba expandir el imperio y el dominio del catolicismo en el siglo XV. En el siglo XXI son otros poderes, las compañías multinacionales, que también vienen de afuera a implantar nuevas formas de someter, de silenciar y perpetuar el discurso del pasado.

Colón, como lo han señalado Lipsett-Rivera y Rivera Ayala, “[...] is not just a historical personage. He is also a cultural icon who carries many burdens. He is alternately a hero $[\ldots]$, the founder of a new race $[\ldots]$ and the person who initiated the devastation of native civilizations" (13).

Con esta fuerte carga semántica, se hace necesario decidir sobre la faceta que se va a representar y en También la lluvia la balanza se inclina hacia la tercera opción: la llegada de Colón con los barcos y la tripulación es catastrófica para la población indígena a causa de la obsesión por el oro, un recurso natural abundante en el nuevo mundo, pero también a causa de otros factores como la barrera lingüística y la concepción del Otro como inferior. En este sentido, resulta acertado recordar los planteamientos de Tzvetan Todorov en su libro The Conquest of America: The Question of the Other (1984). Todorov sostiene que "el descubrimiento" es esencial para nosotros hoy; discute los propósitos pecuniarios de la empresa; propone una función específica de la tierra y de la naturaleza y arguye que el discurso colombino promueve la creación del mito del "buen salvaje". Estos discursos encuentran una vía de expresión en la pantalla. La conceptualización que hace Costa de la población local en su papel de extras es profundamente similar a la que hiciera Colón con respecto a los indígenas. Según Todorov, la población indígena de los textos colombinos es sólo parte del paisaje, de la naturaleza, de la tierra que se puede explotar y por lo tanto no tiene voz. También la lluvia muestra la persistencia de dicha ideología; de igual manera, expone la fiebre del oro en la época de Colón para cuestionar el discurso sobre la conquista y la colonización.

${ }^{11}$ La reseña de Jorge Marí sobre la película hace hincapíe en la conexión con La dolce vita (1960) de Federico Fellini, en la que una escultura de Jesucristo con los brazos abiertos es transportada en helicóptero a la Plaza de San Pedro en Roma. Otra conexión puede hacerse con Cabeza de Vaca (1992), en la cual una enorme cruz es transportada por el desierto al final de la película.

Revista Iberoamericana, Vol. LXXXI, Núm. 251, Abril-Junio 2015, 523-537 ISSN 0034-9631 (Impreso) 
El retrato, o mejor el fantasma onmipresente de Colón se compone de la siguiente manera: en la película dentro de la película, Antón (Elejalde), quien hace el doble papel de Colón y del actor que lo interpreta, manifiesta a Costa sus dificultades para memorizar el libreto y expresa su deseo de también mostrar las dudas y angustias del almirante. Parte del reto para Antón radica en tener que adherirse y producir verbalmente el texto de la primera carta escrita por Colón, pues, aunque adaptado a las formas lingüísticas actuales, el director quiere ser fiel a él. Durante una conversación íntima entre Antón y Costa, Antón manifiesta reserva en cuanto a este plan por considerarlo arriesgado. Dice Antón: "Pues esta es exactamente la primera carta que Colón envió desde el nuevo mundo a la corona española. Por eso Sebastián es tan fiel al texto. No sé si funcionará. Es un poco arriesgado, pero me gusta. Pero si además, me diera un pequeño espacio para presentar al hombre, sea con sus dudas, sus...sus zozobras...".

Aunque Antón no explica por qué considera la adhesión al texto colombino arriesgada, sus palabras sugieren las tensiones que se generan entre el discurso histórico escrito, supuestamente más legítimo, veraz y confiable y su transferencia al medio cinematográfico. Sin embargo, como lo ha afirmado Hayden White en "Historiography and Historiophoty", "No history, visual or verbal, 'mirrors' all or even the greater part of the events or scenes of which it purports to be an account [...]. Every written history is a product of processes of condensation, displacement, symbolization, [...] exactly like those used in [...] filmed representation" (55). Desde esta perspectiva, cabe entonces cuestionar el significado de "ser fiel al texto". Colón no ofrece (no puede ofrecer) fidelidad ni en la carta, ni en el diario por tener éstos motivos ulteriores. Además, si la película se inclinara en favor de serle fiel al texto colombino no habría posibilidad de contar la otra historia y más importante aún: no se podría representar a, ni escuchar la voz de Hatuey, puesto que Colón no hace ninguna referencia a él. Finalmente, También la lluvia se hubiera convertido en una cinta que como 1492: The Conquest of Paradise, representa a Colón como una figura heroica. ${ }^{12}$ No se muestra en la película si hay una negociación entre Antón y Sebastián, puesto que las escenas siguientes retratan a Colón en el papel de opresor y explotador de los indígenas. Para examinar dicha representación, me concentraré en la secuencia comprendida entre los minutos 14:23 a 16:22. La secuencia es importante por dos razones: contiene los atributos que resalta Todorov (el carácter económico de la empresa colombina y el propósito de esclavizar) y en segundo lugar en ella participa Hatuey, figura de singular importancia en la historia de resistencia al imperio español en el Caribe y sin embargo marginal. Sus acciones son poco conocidas y valoradas a pesar de ser considerado el "primer rebelde de América", según lo evidencia el monumento erigido en su honor en Baracoa, Cuba.

12 Dirigida por Ridley Scott, esta película fue estrenada en 1992 para celebrar los quinientos años del primer viaje de Colón. Véase el artículo de Sonia Lipsett-Rivera y Sergio Rivera Ayala.

Revista Iberoamericana, Vol. LXXXI, Núm. 251, Abril-Junio 2015, 523-537 ISSN 0034-9631 (Impreso) ISSN 2154-4794 (Electrónico) 
La escena se filma utilizando una combinación alternada de tres planos: el plano largo, el plano medio y el primer plano. El plano largo permite observar el escenario completo de la secuencia y sus componentes ${ }^{13}$ mientras los otros dos se enfocan en los objetos y especialmente en los personajes y sus relaciones. En primer lugar aparece nuevamente la cruz, enfocada desde el plano picado (de arriba hacia abajo), los árboles, un grupo de niñas y niños indígenas, los conquistadores (de espaldas), un par de caballos $\mathrm{y}$, por último, los indígenas a quienes va dirigido el discurso que pronuncia la voz de Colón. Aunque su presencia no se registra hasta ya avanzada la secuencia, las palabras atribuidas al almirante dominan la escena mientras una voz indígena, cuya fuente aparece también después, las traduce al quechua. La voz de Colón lleva consigo una carga poderosa porque el discurso tiene como propósito desestabilizar la estructura social y económica de la comunidad indígena para crear un grupo social subordinado a la influencia invisible de poderes europeos: el Papa y los Reyes Católicos. Dice Colón: "Solicitamos que aceptéis al Papa y a sus majestades como legisladores del universo".

La voz de Colón invade la escena y se escucha primero. Su discurso es el medio por el cual manifiesta su autoridad e intenciones de doblegar, inicialmente a través de un débil intento de persuación: "a cambio os ofrecemos nuestro amor y nuestra caridad" y posteriomente de amenaza si la comunidad se niega a aceptar los nuevos poderes. Al preguntar Hatuey, a través del traductor, qué pasa si no aceptan, Colón responde: "os haremos esclavos y dispondremos de vosotros a voluntad [...] y os causaremos tanto daño como podamos", enfatizando así su capacidad de infundir miedo y prevenir cualquier tipo de resistencia. Finalmente, el encuentro no tendría el mismo poder sin otro elemento clave: el oro. El intercambio entonces concluye con otra pregunta: “¿qué queréis?" y su correspondiente réplica: "impuestos. Cada indio mayor de catorce años tendrá que llenar un cascabel con oro". No obstante, en una secuencia posterior, el discurso de Colón encuentra las voces de oposición de Bartolomé de las Casas y Antonio de Montesinos. Dicha secuencia incluye el famoso sermón de este último dirigido "a los ricos y poderosos de la isla de Santo Domingo" en el que denuncia la crueldad de la esclavitud indígena, diciendo: "[...] un oro que hace girar la inmensa rueda del comercio [...] Decidme con qué derecho y con qué justicia tenéis en tan cruel y horrible servidumbre a estos indios [...]" (25:20-26:01).

El énfasis en los discursos (Colón, Montesinos, Hatuey) apunta a la primacia de la voz diegética e invierte en cierta medida la jerarquía tradicional entre imagen y sonido. Para explicar dicha inversión, Elsaesser y Hagener en la sección de su libro Film Theory: An Introduction Trough the Senses, dedicada a la acústica y el espacio,

${ }_{13} \mathrm{Al}$ referirse a la cinematografía y en particular a la posición de la cámara, Jordan y Allison precisan que una cinta en la que se incluyen planos largos enfatiza el contexto por encima del drama y la dialéctica sobre la personalidad.

Revista Iberoamericana, Vol. LXXXI, Núm. 251, Abril-Junio 2015, 523-537 ISSN 0034-9631 (Impreso)

ISSN 2154-4794 (Electrónico) 
mencionan las razones que Michel Chion considera relevantes en este caso: "Cinema [...] is an audio-event before it becomes a visual one; [...Chion] stresses the ubiquity and materiality of sound in relation to the image, which crucially depends on the sound to give the image body and substance" (145).

El intercambio entre Hatuey y Colón revela tensiones, debido a que este último busca entronizar la dependencia y la indefensión. La voz de Colón es la voz autoritaria del padre que genera el mito de los indígenas como niños que no tienen voz, representando así el mencionado mito del "buen salvaje". No obstante, el uso del sonido diegético cuestiona la construcción del mito y proyecta la voz de Hatuey como agente de resistencia. Robert Rosenstone observa en el proceso de ver y escuchar voces subalternas en la pantalla un cuestionamento de la historiografía tradicional para la cual "a truthful past can only be told in words on the page" (5). ${ }^{14}$

La persistencia de los abusos agudizan mucho más el ímpetu de rebelión en Hatuey, quien junto con otros hombres, en una de las escenas de mayor dramatismo de la cinta, es quemado vivo, atado a una cruz en una ejecución pública. En esta sección de la película Bollaín se basa en uno de los relatos existentes sobre esta figura, especialmente el de Bartolomé de las Casas en la Brevísima relación de la destrucción de las Indias. Allí se apunta que antes de morir Hatuey rechaza la fe cristiana y prefiere ir al infierno. ${ }^{15} \mathrm{La}$ escena concluye con los gritos desesperados de Hatuey y otros doce crucificados, que descontrolan y atemorizan a los perpetradores. Las voces y los gritos desbordan el control y la autoridad de los capitanes (Colón no está presente), porque invaden el espacio e intensifican la injusticia del sacrificio; además, producen emociones de identificación y empatía en los espectadores. En suma, retomando las ideas de Chion, provocan una agitación de amplias proporciones: la multitud de indígenas, hombres, mujeres, niñas y niños (extras) que ha sido obligada a presenciar la ejecución no permanece en silencio; une sus voces a las de los crucificados para gritar al unísono, de forma atronadora el nombre de Hatuey. Esta maniobra subvierte, al interior de la cinta, los presupuestos que planteara Costa en cuanto al papel de los extras como "paisaje humano". Bollaín destaca así el enorme poder de la voz y contribuye de igual manera a recuperar el legado de insubordinación indígena. Vale la pena agregar que También la lluvia es la primera película que se realiza sobre Hatuey.

\footnotetext{
${ }^{14}$ Este argumento se alinea con el presentado en La historia oficial del argentino Luis Puenzo (1985).

${ }^{15}$ Aquí sigo el texto de Las Casas publicado en la página web de José María Ruano de la Haza que dice: "Atado a un palo decíale un religioso de San Francisco, santo varón que allí estaba, algunas cosas de Dios y de nuestra fe, (el cual nunca las había jamás oído), lo que podía bastar aquel poquillo tiempo que los verdugos le daban, y que si quería creer aquello que le decía iría al cielo, donde había gloria y eterno descanso, y si no, que había de ir al infierno a padecer perpetuos tormentos y penas. Él, pensando un poco, preguntó al religioso si iban cristianos al cielo. El religioso le respondió que sí, pero que iban los que eran buenos. Dijo luego el cacique, sin más pensar, que no quería él ir allá, sino al infierno, por no estar donde estuviesen y por no ver tan cruel gente" (Casas).
}

Revista Iberoamericana, Vol. LXXXI, Núm. 251, Abril-Junio 2015, 523-537 ISSN 0034-9631 (Impreso) ISSN 2154-4794 (Electrónico) 
Al tratarse de una película dentro de otra, la secuencia anterior concluye con el ya familiar "corte" verbalizado por el director. La oscilación entre los planos espaciotemporales permite que Hatuey pronto aparezca en su papel de Daniel, quien simboliza por lo tanto el retorno del discurso silenciado. Daniel es un activista comprometido con la causa de traer agua (yaku, en quechua) a su comunidad y preservarla de las fuerzas estatales y multinacionales que la quieren privatizar. Varios segmentos de la película muestran el paulatino ascenso de Daniel dentro del movimiento: primero es parte del grupo que construye una suerte de acueducto; luego se desempeña como organizador de reuniones comunitarias para tomar decisiones en cuanto al tipo de acciones colectivas que se van a realizar, y finalmente, como vocero en manifestaciones tanto en la sede de "Aguas de Bolivia" como frente a la alcaldía de Cochabamba. A pesar de que Costa le exija que por beneficio de la película se mantenga al margen del conflicto por el agua, y para lograrlo le dé dinero, Daniel no le hace caso. El legendario Hatuey y el hombre que lo representa se funden en uno solo, quien busca redefinir la historia y reivindicar su posición y la de la mayoría de sus compatriotas, marginados, explotados y engañados por el discurso de los poderes centrales, que operan dentro y fuera de Bolivia. Mientras ya no es necesario llenar un cascabel con oro para llenar las arcas de Colón, los reyes o sus obispos, ahora el gobierno municipal de Bolivia exige aceptar la ley (2029) ${ }^{16}$ que, entre otras limitaciones, prohibe recolectar el agua de la lluvia (de ahí el título de la película) para consolidar el proceso de privatizar el servicio. ${ }^{17}$

La intensa lucha del presente por detener la privatización y refutar el discurso que la acompaña, refuerza la noción de la circularidad del tiempo. El presente parece repetir el pasado, los actores son nuevos, en este caso un consorcio multinacional, International Waters Limited (británico) y Bechtel (estadounidense) aludido por Daniel en uno de sus discursos enfrente de "Aguas de Bolivia" al mencionar "una compañía cuyos propietarios están en Londres y California". En el siglo XXI el agua sustituye al oro como objeto que generará ganancias para la entidad que la proveerá a un precio exhorbitante $(\$ 450$ al año a cada usuario, según la cinta) que la mayoría de cochabambinos no podrá pagar. Igualmente, la película muestra que, como el oro, el agua potable es hoy en día un recurso cada vez más escaso y de difícil acceso. Por lo tanto, la comunidad la protege constantemente no solamente con cadenas y candados, sino también levantando la voz para frenar a los representantes uniformados del gobierno que llegan a apropiarse de un pozo que la colectividad ha cavado. En la secuencia casi documental y comprendida entre los minutos 22 a 24, una invisible línea horizontal divide al grupo de mujeres que se enfrenta a los representantes del municipio. Sin embargo, poco a poco, la línea

16 Veáse http://www.lexivox.org/norms/BO-L-2029.xhtml

${ }^{17}$ La privatización, implementada a insistencia del Banco Mundial condujo al gobierno a realizar contratos con Aguas del Tunari, subsidiaria de International Waters Ltd, 50\% propiedad de la firma Bechtel, corporación mundialmente conocida por realizar trabajos de ingeniería.

Revista Iberoamericana, Vol. LXXXI, Núm. 251, Abril-Junio 2015, 523-537 
divisoria se borra, en una lucha cuerpo a cuerpo, que unida al discurso de las mujeres, basado en su identidad de madres, contiene la autoridad de los hombres, la desborda y les advierte: "vamos a luchar hasta las últimas consecuencias". En este punto vuelven a ser relevantes las ideas de Hayden White en cuanto a que el cine y el video "are better suited than written discourse to the actual representation of certain kinds of historical phenomena-landscape, scene, atmosphere, [...] battles, crowds and emotions" (53). Las emociones y tensiones expresadas tanto por la imagen como por el discurso transmiten además el comportamiento "verbocéntrico" a que se refiere Chion, que resulta más difícil de comunicar en un texto escrito. Por otro lado, la escena mencionada contribuye al cuestionamiento de los discursos del poder: "Los señores aquí están haciendo su trabajo" dice uno de los hombres; a lo cual una de las mujeres responde: “quitarnos el agua?"

La volátil situación generada por el plan estatal para la privatización del agua desemboca en otras protestas enfrente de la alcaldía en el centro de Cochabamba, mientras el equipo de filmación es recibido por el alcalde y atendido con gran deferencia y con champán. Aunque Costa, Sebastián y el resto del equipo del rodaje asumen de nuevo la posición de observadores ubicados en lo alto del edificio desde donde miran a la multitud en movimiento en el plano inferior, reivindican en diálogo con el alcalde las exigencias de los cochabambinos concentrados afuera.

Durante la cordial conversación (minutos 50:20 a 52:09) Sebastián expresa: "Bueno, espero no le importe que lo diga, pero la verdad me parece bastante razonable lo que ellos piden". El alcalde, a su vez, atribuye las ideas de Sebastián a falta de información y afirma: "Somos un país con escasos recursos. Nos es muy difícil mantener abastecimiento de agua sin una fuerte inversión extranjera. Dada su larga historia de explotación, los indios llevan la desconfianza en los genes. Se hace muy difícil razonar con ellos, cuando además son analfabetos. Pero, es así. Tenemos informes objetivos de profesores de Harvard, del FMI [...]". ${ }^{18}$ Es decir, vuelve a esgrimir el argumento de los indígenas como inferiores, como personas que no tienen derecho a hablar y por lo tanto están inevitablemente supeditados a aceptar el dicurso del poder. Las ideas del alcalde coinciden con las características que Foucault ha establecido en cuanto al discurso del poder, ya que "The same discourse, characteristic of the way of thinking or the state of knowledge at any one time [...] will appear across a range of text, and as forms of conduct, at a number of different institutional sites within society" (72-73). En la escala de valores manejada por el gobierno local, el discurso supuestamente objetivo que viene de afuera, producido por centros académicos de prestigio como la Universidad de Harvard y el FMI no está influido por intereses políticos o económicos y por lo tanto tiene más

18 Esta frase puede referirse a Jeffrey Sachs, una de las "luminarias" del departamento de economía de Harvard y quien en los años 80 estuviera encargado de desarrollar un plan anti-inflacionario para Bolivia, aunque según Naomi Klein, "knew next to nothing about Bolivia and its long history of colonial exploitation" (The Shock Doctrine 171).

Revista Iberoamericana, Vol. LXXXI, Núm. 251, Abril-Junio 2015, 523-537 ISSN 0034-9631 (Impreso)

ISSN 2154-4794 (Electrónico) 
credibilidad y peso que el que están tratando de articular las personas directamente afectadas por el costo de la privatización. Por otro lado, la burda defensa del FMI es completamente incoherente con el discurso que promueve esta entidad con respecto a la reducción de la pobreza en Bolivia: cobrar cuotas imposibles por el servicio de agua en vez de aliviar la pobreza, tendrá el efecto contrario de intensificarla. ${ }^{19}$

Irónicamente, Antón, el actor que representa a Colón, interrumpe inmediatamente el argumento del alcade diciendo: "me encantaría saber cómo se las arreglarían estos cabrones del FMI para mantener a sus familias con 40 putos dólares al mes, ¿que no?" La respuesta del alcalde a este comentario es la siguiente: "en este mundo globalizado los indios se dedican a quemar los recibos de agua y a tirar piedras a la policía. Es el victimismo contra la modernidad". Este discurso ahonda el conflicto en términos de quien tiene espacio representativo mientras los que protestan afuera gritan: "Fusil, metralla, el pueblo no se calla". Costa manifiesta su preocupación, pero su discurso tiene un tono conciliador. Antes de que el alcalde se retire a ocuparse de la "pequeña trifulca doméstica", Costa le dice: "imagino que están tratando de llegar a un acuerdo". A lo que el alcalde replica: "Si cedemos un centímetro estos indios nos llevarán de nuevo a la edad de piedra”.

Esta posición inflexible y racista conduce a enfrentamientos mucho más violentos con las fuerzas del orden: una huelga general con bloqueo de carreteras, lanzamiento de petardos y piedras, además de heridos y detenciones que paralizaron la ciudad. Después de la revuelta, la cámara nos muestra una toma panorámica de Cochabamba que sirve de cierre a una secuencia en la que Costa imagina a un hombre quien vestido de sacerdote camina mientras hace sonar una campana diciendo "detengan la lucha, el agua es de ustedes". Estas palabras, en cierto modo, legitimizan la victoria de la población, recuerdan y reivindican la labor en favor de los indígenas que llevaron a cabo Bartolomé de Las Casas y Antonio de Montesinos. Es un día soleado y el encuadre en ángulo en picado sugiere que el orden y la tranquilidad parecen haberse restaurado en la ciudad. Este tipo de encuadre implica, según Ryan y Lenos, que alguien o algo ha perdido poder. En este caso, la pérdida es para muchos: el gobierno de la ciudad, el consorcio multinacional, el equipo de filmación y para el mismo Daniel quien estuvo a punto de perder a su hija. Cochabamba se muestra como un espacio metafóricamente liberado del poder que las alianzas económico-políticas de fuerzas internas y externas querían ejercer sobre la población más vulnerable. En cuanto al equipo de filmación la pérdida se traduce en la imposibilidad de terminar la película, en no contar su final. No obstante, el proyecto truncado alude a la imposibilidad de dar final a una historia que no lo tiene. La maniobra da libertad para que el discurso sobre "lo que pasó" siga

19 Veáse los documentos contenidos en la página del FMI con respecto a Bolivia, en particular los fechados enero 13, 2000; marzo, 31 y mayo 21, 2001.

\footnotetext{
Revista Iberoamericana, Vol. LXXXI, Núm. 251, Abril-Junio 2015, 523-537 
circulando y se siga contando desde otras perspectivas. La vertiginosa secuencia final de la película así lo sugiere. Además, la última palabra expresada por Costa, quien aparece en esta secuencia final, es yaku, agua, una palabra que contiene ahora una multitud de significados, de acuerdo a quien la utilice. Sin embargo, es un emblema poderoso de la victoria de la gente de Cochamba.

El protagonismo social de Daniel, unido al de muchos otros se traduce en decisiones institucionales. Costa le enseña a Daniel el titular divulgado en una publicación llamada El Correo del Sur, que declara: "Multinacional abandona Bolivia después de la guerra del agua". De hecho, el gobierno boliviano se vio obligado a dar marcha atrás y a cambiar su discurso al anunciar la cancelación del contrato con el consorcio multinacional. Este resultado manifiesta la crisis de un sistema injusto de discursos (tratados) internacionales y la influencia nefasta de las corporaciones cuyo interés en la alta rentabilidad de su inversión, asociado con el desconocimiento de la población local, su historia, su lengua y sus peculiaridades, es incompatible con el derecho de la población al agua. Las guerras del agua situaron a Bolivia, un país periférico y a Cochabamba y sus habitantes marginales en el centro de las luchas por el control y la utilización equitativa de recursos vitales. Sirvieron otro fin de gran relevancia: re-escribir el discurso oficial de la historia desde la perspectiva de los afectados. Permitieron, como señalara Foucault, la emergencia de otro discurso en otro momento histórico para desbancar el discurso vigente y producir otras dinámicas en cuanto a las relaciones de poder. La técnica cinematográfica subraya la importancia de conectar los discursos del pasado con los del presente para educar y para mostrar la importancia de cuestionarlos. El sonido diegético funciona, por su parte, para apoyar dicha conexión y para amplificar la potencia del discurso (la voz) de personajes como Hatuey/Daniel.

Para dar respuesta a las preguntas formuladas en la parte incial de este artículo, También la lluvia es una cinta que nos permite escuchar las voces de quienes se encuentran socialmente marginados ya sea por razones económicas o de distancia de los centros de poder e influencia. Por otra parte, la cinta contribuye a observar de cerca el modus operandi del sistema capitalista en el que el agua (y aún la lluvia) se puede controlar, comprar y vender a buen precio, privando de ella a quienes no la pueden pagar. En este sentido Bollaín produce una suerte de crónica sobre la crítica situación de Bolivia a comienzos del milenio y las acciones radicales de sus habitantes para proteger su derecho no sólo al discurso, sino también su derecho a un bien básico.

De igual manera, la directora aprovecha las circunstancias que rodearon la producción de su película para mostrar cómo los recursos de América han sido utilizados para la ganancia comercial de unos pocos privilegiados, incluido tal vez su propio equipo de filmación. ${ }^{20}$ La directora evidencia en esta película su talento para exponer la profundidad

${ }^{20}$ Durante la conversación con el alcalde, Sebastián admite que los extras reciben \$2 dólares por su trabajo. Antón, por su parte, afirma en otra sección de la cinta que le pagan dos millones de bolivianos,

Revista Iberoamericana, Vol. LXXXI, Núm. 251, Abril-Junio 2015, 523-537 ISSN 0034-9631 (Impreso)

ISSN 2154-4794 (Electrónico) 
histórica de la injusticia, pero también para ilustrar a través de Costa que es posible evolucionar, de pasar de un estado de ceguera moral y de interés puramente material a expresar solidaridad con quienes eran considerados inferiores. De hecho, Costa decide dejar el proyecto de filmación para ayudar a encontrar a Belén, la hija de Daniel, quien ha resultado herida durante los bloqueos.

Daniel, por su parte, consigue pasar de la subordinación a la autonomía; logra producirse a sí mismo, aunque tiene que pagar un precio. Por esa razón sus palabras resuenan aún después de salir de la sala de cine: "siempre nos cuesta tan caro. Nunca es fácil. Ojalá hubiese otra forma, pero no la hay”. Y a la pregunta de Costa, “¿y tú, Daniel, qué vas a hacer?", Daniel responde: "Sobrevivir como siempre. Es lo que hacemos mejor".

\section{OBRAS CITADAS}

Cabeza de Vaca. Nicolás Echavarría, dir. Channel Four Films, 1991.

Casas, Bartolomé de las. Brevísima relación de la destrucción de las Indias. $<\mathrm{http}: / /$ aix1.uottawa.ca/ jmruano/relacion.pdf>. 2 julio 2013.

Chion, Michel. Audio-Vision. Sound on Screen. Claudia Gorman, ed. y trad. Nueva York: Columbia UP, 1990.

Colón, Cristóbal. “Carta a D. Luis de Santángel”. Biblioteca Virtual Miguel de Cervantes. $<$ http://www.cervantesvirtual.com/obra-visor/relaciones-y-cartas-de-cristobalcolon--0/html/>. 26 junio 2013.

"Relación del primer viaje del descubrimiento". Relaciones y cantos de Cristóbal Colón. Biblioteca Virtual Miguel de Cervantes. < http://www.cervantesvirtual.com/ obra-visor/relaciones-y-cartas-de-cristobal-colon--0/html/>. 26 junio 2013.

Elsaesser, Thomas y Malte Hagener. Film Theory: An Introduction Through the Senses. Nueva York: Routledge, 2010.

Flores de otro mundo. Icíar Bollaín, dir. Producciones La Iguana, 1999.

Fondo Monetario Internacional. <http://www.lexivox.org/norms/BO-L-2029.xhtml>. 2 julio 2013.

Hall, Stuart. "Foucault: Power, Knowledge and Discourse". Discourse Theory and Practice: A Reader. Margaret Weatherell, Stepahnie Taylor y Simeon J. Yates, eds. Londres: Sage Publications, 2001. 72-81.

Jordan, Barry y Mark Allison. Spanish Cinema: A Student's Guide. Londres: Hodder y Arnold, 2005.

(aproximadamente \$279.788 dólares, según Oanda http://www.oanda.com/) una suma extravagante en comparación.

Revista Iberoamericana, Vol. LXXXI, Núm. 251, Abril-Junio 2015, 523-537 ISSN 0034-9631 (Impreso) 
Klein, Naomi. The Shock Doctrine: The Rise of Disaster Capitalism. Canadá: Alfred A. Knopf, 2007.

La dolce vita. Federico Fellini, dir. Pathé Cinema, 1960.

Landy, Marcia. "The Historical Film: History and Memory in Media." The History on Film Reader. Marnie Hughes-Warrington, ed. Londres: Routledge, 2009. 42-52.

La historia oficial. Luis Puenzo, dir. Cinemanía, 1985.

Land and Freedom. Ken Loach, dir. Paralax Pictures, 1995.

Lipsett-Rivera, Sonya y Sergio Rivera Ayala. "Columbus Tales On the Faces ofDarkness; of Film and Historical Myth in 1492: The Conquest of Paradise." Based on a True Story: Latin American History of the Movies. Donald Pithian Stevens. Lanham, MD: SR Books, 2007. 13-28.

Marí, Jorge. "También la lluvia (reseña)". Hispania 95/2 (2012): 369-371. Project MUSE. <http://muse.jhu.edu/>. 3 jul. 2013.

Mataharis. Icíar Bollaín, dir. Producciones La Iguana, 2007.

Rosenstone, Robert A. History on Film/Film on History. Harlow, UK: Pearson y Longmang, 2006.

Ryan, Michael y Melissa Lenos. An Introduction to Film Analysis. Technique and Meaning in Narrative Film. Nueva York: Continuum Books, 2012.

1492: The Conquest of Paradise. Ridley Scott, dir. Paramount Pictures, 1992.

También la lluvia. Icíar Bollaín, dir. Morena Films, 2010.

Te doy mis ojos. Icíar Bollaín, dir. Alta Producción, 2003.

Todorov, Tzvetan. The Conquest of America. The Question of the Other. Richard Howard, trad. Nueva York: Harper \& Row, 1984.

White Hayden. "Historiography and Historiopothy." The History on Film Reader. Marnie Hughes-Warrington, ed. Londres: Routledge, 2009. 53-59.

Zinn, Howard. A People's History of the United States: 1492 to the present. Nueva York: Harper Perennial, 1995. 
